\title{
Phylogeny of Metschnikowia Species Estimated from Partial rRNA Sequences
}

\author{
L. C. MENDONÇA-HAGLER, ${ }^{1 *}$ A. N. HAGLER, ${ }^{1}$ AND C. P. KURTZMAN ${ }^{2}$ \\ Instituto de Microbiologia, Universidade Federal do Rio de Janeiro, Ilha do Fundao, Rio de Janeiro, RJ \\ 21941, Brazil, ${ }^{1}$ and Microbial Properties Research, National Center for Agricultural Utilization Research, \\ Agricultural Research Service, U.S. Department of Agriculture, Peoria, Illinois $61604^{2}$
}

\begin{abstract}
Phylogenetic relationships of species assigned to the genus Metschnikowia were estimated from the extents of divergence among partial sequences of rRNA. The data suggest that the aquatic species (Metschnikowia australis, Metschnikowia bicuspidata, Metschnikowia krissii, and Metschnikowia zobellii) and the terrestrial species (Metschnikowia hawaiiensis, Metschnikowia lunata, Metschnikowia pulcherrima, and Metschnikowia reukaufii) form two groups within the genus. $M$. lunata and $M$. hawaiiensis are well separated from other members of the genus, and $M$. hawaiiensis may be sufficiently divergent that it could be placed in a new genus. Species of the genus Metschnikowia are unique compared with other ascomycetous yeasts because they have a deletion in the large-subunit rRNA sequence that includes nucleotides 434 to 483.
\end{abstract}

The ascomycetous yeast genus Metschnikowia is defined by the presence of multilateral budding of vegetative cells and by the production of one or two needle-shaped ascospores in elongate asci. There are relatively few differences in physiological traits among the species of the genus (21). Species isolated from terrestrial habitats are typically associated with flowers or fruits and insect vectors, and species isolated from aquatic habitats are often parasitic in invertebrates but can also be isolated as free-living forms in water. Isolations of Metschnikowia species have been sporadic, but the unusual needlelike ascospore morphology of these organisms and their associations with other organisms have attracted the attention of taxonomists and ecologists. Metschnikoff (20) first described a species of this group as Monospora bicuspidata and also noted the function of its needle-shaped spores in the parasitic invasion of Daphnia magna. The name Metschnikowia was introduced by Kamienski in 1899 (9), and descriptions of anamorphs of two terrestrial Metschnikowia species, Candida pulcherrima (16) and Candida reukaufii (7), followed. Taxonomic work on this genus was stimulated by the isolation of the aquatic species Metschnikowia zobellii and Metschnikowia krissii reported in 1961 (30), of Metschnikowia bicuspidata (the type species) in 1964 (32), and of additional aquatic strains of Metschnikowia species by Spencer et al. (28) and Fell and Hunter (2). Needle-shaped ascospores were observed in strains of $C$. pulcherrima and $C$. reukaufii, showing that these organisms are members of the genus Metschnikowia $(2,24)$. In vivo hybridization experiments defined an aquatic group of three species and a predominantly terrestrial chlamydospore-forming group containing two species $(4,24$, 25). Metschnikowia lunata, another chlamydospore-forming species, was isolated from flowers and differed from other Metschnikowia species in having lunate vegetative cells (6). DNA reassociation studies clarified the delineation of species belonging to the aquatic group. $M$. bicuspidata, $M$. zobellii, and $M$. krissii were shown to be distinct from Metschnikowia australis, and $M$. australis was shown to be a sibling species of $M$. bicuspidata (19). M. zobellii exhibited slightly higher levels of DNA relatedness to the aquatic

\footnotetext{
* Corresponding author.
}

species than to terrestrial taxa, although the interspecific values were $15 \%$ or less.

A yeast that formed unusually large asci and needleshaped ascospores was isolated from flowers and Drosophila sp. and was described as Metschnikowia hawaiiensis by Lachance et al. (14). Although this new species had a terrestrial origin, it did not form chlamydospores, occurred in nature in the haploid condition, and was shown to differ from Metschnikowia reukaufii and Metschnikowia pulcherrima in DNA base sequence complementarity. The lack of chlamydospores suggests that $M$. hawaiiensis could be more closely related to the aquatic group. DNA relatedness data and genetic hybridization data have clearly defined eight species in the genus Metschnikowia. These taxa can be separated into a group of four species that are predominantly associated with aquatic environments and a second group of four species that are associated predominantly with terrestrial environments.

Comparisons of levels of DNA relatedness allow good separation of strains at the species and sibling species level, but do not allow resolution of relationships above this level (10). rRNA sequence comparisons have been used to determine phylogenetic relationships above the species level (33). At this time complete sequencing of small- and large-subunit rRNAs is impractical for comparisons of large numbers of strains, but partial sequences of bacterial rRNAs were found by Lane et al. (15) to yield phylogenetic trees similar to those derived from complete sequences. For yeasts, partial sequencing of $18 \mathrm{~S}$ and $25 \mathrm{~S}$ rRNAs having different degrees of conservation has allowed resolution of species relationships in several genera $(3,8,12,13,22,23,34,35)$. In an effort to better understand species relationships in the genus Metschnikowia, we determined partial rRNA sequences for the type strains of all of the species that are assigned to this genus at this time.

\section{MATERIALS AND METHODS}

Yeast strains. The strains of Metschnikowia species and the comparative taxa used are listed in Table 1. All of these strains are maintained in the Agricultural Research Service Culture Collection, National Center for Agricultural Utilization Research, Peoria, Ill.

rRNA extraction and purification. Cells were grown at 
TABLE 1. Strains examined and selected phenotypic characteristics of Metschnikowia species and reference species

\begin{tabular}{|c|c|c|c|c|c|}
\hline \multirow{2}{*}{ Species } & \multicolumn{2}{|c|}{ Strain designations ${ }^{a}$} & \multirow{2}{*}{ Habitat } & \multirow{2}{*}{$\begin{array}{l}\text { No. of spores } \\
\text { per ascus }\end{array}$} & \multirow{2}{*}{ Chlamydospores } \\
\hline & NRRL & CBS & & & \\
\hline M. australis (Fell and Hunter) Mendonça-Hagler et al. & $\begin{array}{c}\mathrm{Y}-17414^{\mathrm{T} b}, \\
\mathrm{Y}-7014\end{array}$ & $\begin{array}{r}5847^{\mathrm{T}} \\
5848\end{array}$ & Aquatic & 2 & - \\
\hline M. bicuspidata (Metschnikoff) Kamienski & $\mathrm{YB}^{-} 4993^{\mathrm{T}}$ & $5575^{\mathrm{T}}$ & Aquatic & 2 & - \\
\hline M. hawaiiensis Lachance et al. & Y-17272 & $7432^{\mathrm{T}}$ & Terrestrial & 2 & - \\
\hline M. krissii (van Uden and Castelo-Branco) van Uden & $\mathrm{Y}-5389^{\mathrm{T}}$ & $4823^{\mathrm{T}}$ & Aquatic & 1 & - \\
\hline M. lunata Golubev & $\mathrm{Y}-7131^{\mathrm{T}}$ & $5946^{\mathrm{T}}$ & Terrestrial & 2 & + \\
\hline M. pulcherrima Pitt and Miller & $\begin{array}{r}\mathrm{Y}-7111^{\mathrm{T}}, \\
\mathrm{Y}-7275\end{array}$ & $5833^{\mathrm{T}}$ & Terrestrial & 2 & + \\
\hline M. reukaufii (Pitt and Miller) & $\mathrm{Y}-7112^{\mathrm{T}}$ & 5834 & Terrestrial & 2 & + \\
\hline M. zobellii (van Uden and Castelo-Branco) van Uden & $Y-5387^{T}$ & $4821^{\mathrm{T}}$ & Aquatic & 1 & - \\
\hline S. cerevisiae Meyen ex Hansen & $Y-12632^{T}$ & $1171^{\mathrm{T}}$ & & & \\
\hline D. hansenii (Zopf) Lodder and Kreger-van Rij & $Y-7426^{\mathrm{T}}$ & $767^{\mathrm{T}}$ & & & \\
\hline
\end{tabular}

${ }^{a}$ NRRL, Agricultural Research Service Culture Collection, Peoria, Ill.; CBS, Centraalbureau voor Schimmelcultures, Delft, The Netherlands.

${ }^{b} \mathrm{~T}=$ type strain.

$25^{\circ} \mathrm{C}$ in $\mathrm{YM}$ liquid medium (31) on a rotary shaker at $200 \mathrm{rpm}$ for approximately $16 \mathrm{~h}$ and were harvested by centrifugation. The cells were suspended in $2 \times$ SSE buffer (29) at a concentration of $1 \mathrm{~g}$ (wet weight) per $10 \mathrm{ml}$ of buffer; an equal volume of $80 \%$ phenol in water containing $0.1 \%$ 8-hydroxyquinoline, $2 \%$ (vol/vol) dimethyl sulfoxide, and $2 \%$ (vol/vol) xylene was added to this buffer as described by Kurtzman and Liu (12). The cells in the mixture were broken with a Braun cell homogenizer by using 0.5-mm-diameter glass beads. The suspension of broken cells was transferred to a flask and was made $1 \mathrm{M}$ in sodium perchlorate and $1 \%$ in $N$-lauroyl sarcosine. An equal volume of chloroformisoamyl alcohol $(24: 1, \mathrm{vol} / \mathrm{vol})$ was added to the suspension, and the mixture was emulsified by swirling and then placed in an ice bath on a reciprocating shaker for $30 \mathrm{~min}$. The emulsion was separated by centrifugation for $20 \mathrm{~min}$ at $20,000 \times g$, and the top aqueous layer containing rRNA was removed with a pipette for further purification by using the method of Chirgwin et al. (1). The physical integrity of purified rRNA was assessed by performing nondenaturing agarose gel electrophoresis.

Sequencing reactions and sequence comparisons. Sequencing of rRNA was accomplished by using specific oligonucleotide primers and the dideoxynucleotide chain termination method $(15,27)$. Two regions were sequenced from the large (25S) subunit, and one region was sequenced from the small (18S) subunit. The large-subunit primers (and first bases of the rRNA sequences copied) were as follows: 5'-GGTC CGTGTTTCAAGACGG (base 635) and 5'-TTGGAGACCT GCTGCGG (base 1841). The small-subunit primer and first base of the rRNA sequence copied were 5 -ACGGGCGGT GTGTAC and base 1627, respectively. The nucleotide numbers given below are equivalent to the nucleotide numbers in the primary structure of Saccharomyces cerevisiae $(5,18$, 26 ), but the numbers in the aligned sequences differ somewhat from the numbers in the $S$. cerevisiae sequence because of insertions and deletions in the other species. For easier reference below, the three primers described above are designated $25 \mathrm{~S}-635,25 \mathrm{~S}-1841$, and $18 \mathrm{~S}-1627$, respectively. The primers were made with an Applied Biosystems model 381A DNA synthesizer. The nucleotide fragments generated in the chain termination reactions were separated on $8 \%$ acrylamide- $8 \mathrm{M}$ urea gels and were visualized by autoradiography.

Sequences were manually aligned, and dendrograms of the sequence data were calculated by using each of the following three methods: DNAML, version 3.42 , a phylogeny inference, maximum-likelihood program (J. Felsenstein, University of Washington, Seattle); PAUP, version 2.4.1, a parsimony analysis program (D. Swofford, Illinois Natural History Survey, Champaign); and the neighbor-joining method, in which the reproducibility of tree nodes was analyzed by using a bootstrapping program (PHYLIP, version, 3.42; J. Felsenstein). For the initial data treatment we used the maximum-likelihood option. A total of 100 bootstrap trees were generated and examined.

Nucleotide sequence accession numbers. The GenBank accession numbers for $18 \mathrm{~S}$ rRNA segment 1627 and the $25 \mathrm{~S}$ rRNA segments 635 and 1841 are, respectively, L10684, L10646, and L10743 for $M$. australis; L10685, L10644, and L10744 for M. bicuspidata; L10686, L10647, and L10745 for M. hawaiiensis; L10687, L10648, and L10746 M. krissii; L10688, L10650, and L10747 for M. lunata; L10689, L10679, and L10748 for $M$. pulcherrima; L10690, L10680, and L10749 for $M$. reukaufii; L10691, L10681, and L10750 for $M$. zobellii; L10682, L10643, and L10741 for S. cerevisiae; and L10683, L10649, and L10742 for D. hansenii.

\section{RESULTS AND DISCUSSION}

Aligned sequences for the three rRNA segments which were examined are shown in Fig. 1 through 3. The most notable feature of these sequences is a large deletion in the 25S-635-initiated region (nucleotides 434 to 483 ) that is characteristic of all Metschnikowia species but is not found in other genera of ascomycetous yeasts $(11-13,17,22)$. This deleted area is somewhat larger for $M$. lunata than it is for the majority of Metschnikowia species, and it is still larger for $M$. hawaiiensis. A few much smaller deletions are found elsewhere among the sequences.

The nucleotide differences in the highly variable $25 \mathrm{~S}-635$ region are sufficient to separate all of the Metschnikowia species. Comparisons with other yeast genera have shown that the strains of a species either have identical sequences in this region or differ by no more than 2 nucleotides among the approximately 300 nucleotides examined (11). In this study additional strains of $M$. australis and $M$. pulcherrima were compared, and for each species the sequences were identical to the sequence of the type strain.

A matrix of the nucleotide differences among Metschnikowia spp. is shown in Table 2. The extent of nucleotide divergence within the three regions which were sequenced is 


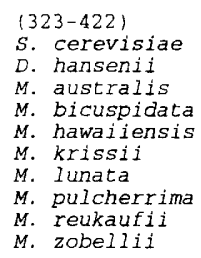

(423-522)

$S$. cerevisiae

$D$. hansenii

$M$. australis

$M$.
$M$. hicuspidata

$M$. krissii

$M$. Iunata

M. pulcherrima

M. reukaufii

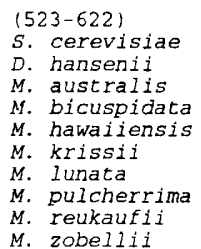

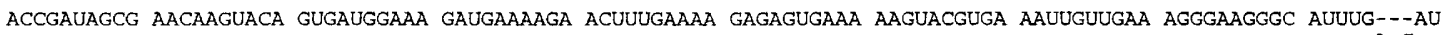

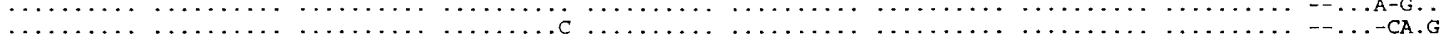

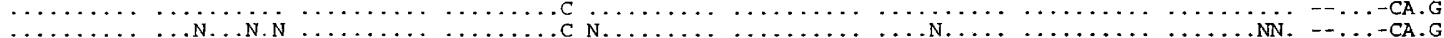

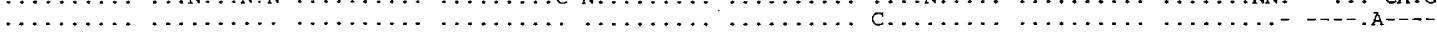

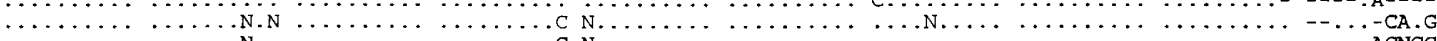

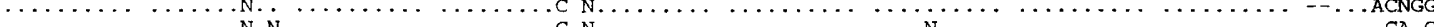

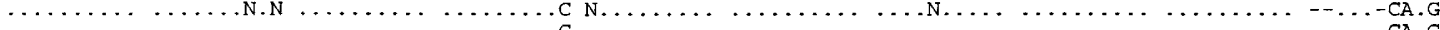

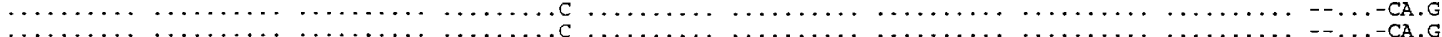

CAGACA--UG GUGUUUUGUG CCCUCUGCUC CUUGUG-GGU AGGGGAAUCU CGCAUUUCAC UGGGCCAGCA UCAGUUUUGG -UGGCAGGAU AAAUCCAUAG

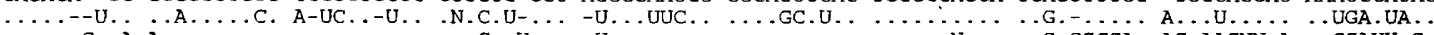

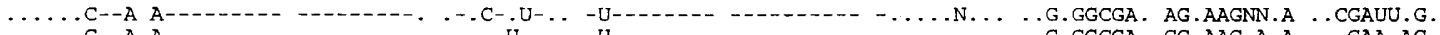
$\ldots \ldots$. C-A A-...... .... . C-A A A-. N. $\ldots \ldots$. CU.A A-1. A .... С . А A-

FIG. 1. Alignment of the 25S rRNA partial sequences initiated at position 635 from Metschnikowia species and the reference taxa $S$. cerevisiae and $D$. hansenii. The sequence of $S$. cerevisiae is given in its entirety for this region and serves as a reference, but there is a departure from correct numbering in this and the other regions examined because of insertions and deletions that occur in the taxa compared. The dots indicate nucleotides identical to nucleotides in the reference species; the dashes indicate that no base is present at a position; and $\mathrm{N}$ indicates an unidentified base.

greater than the extent of divergence found in any other ascomycetous yeast genus examined so far $(12,13,17,22)$. When $M$. hawaiiensis is removed from consideration, the remaining species still constitute a genus that is highly divergent, but one that is comparable in variety to the genera Issatchenkia, Schizosaccharomyces, and Williopsis. In contrast, the levels of species nucleotide divergence in the genera Debaryomyces, Lipomyces, Saccharomyces, and Saturnospora are about one-half the levels in the genera Issatchenkia, Schizosaccharomyces, and Williopsis. The variability observed in this study raises the question of whether the species that are currently assigned to the genus Metschnikowia are all members of the same genus. In the studies cited above, each genus was characterized by unique

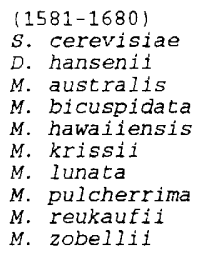

(1681-1780)
S. cerevisiae
D. hansenii
M. australis
M. bicuspidata
M. hawailensis
M. krissii
M. Iunata
M. pulcherrima
M. reukaufii
M. zobellii

(1781-1836)

S. cerevisiae

D. hansenit

M. australis

M. bicuspidata

M. krissii

M. Iunata

pulcherrima

reukaufis
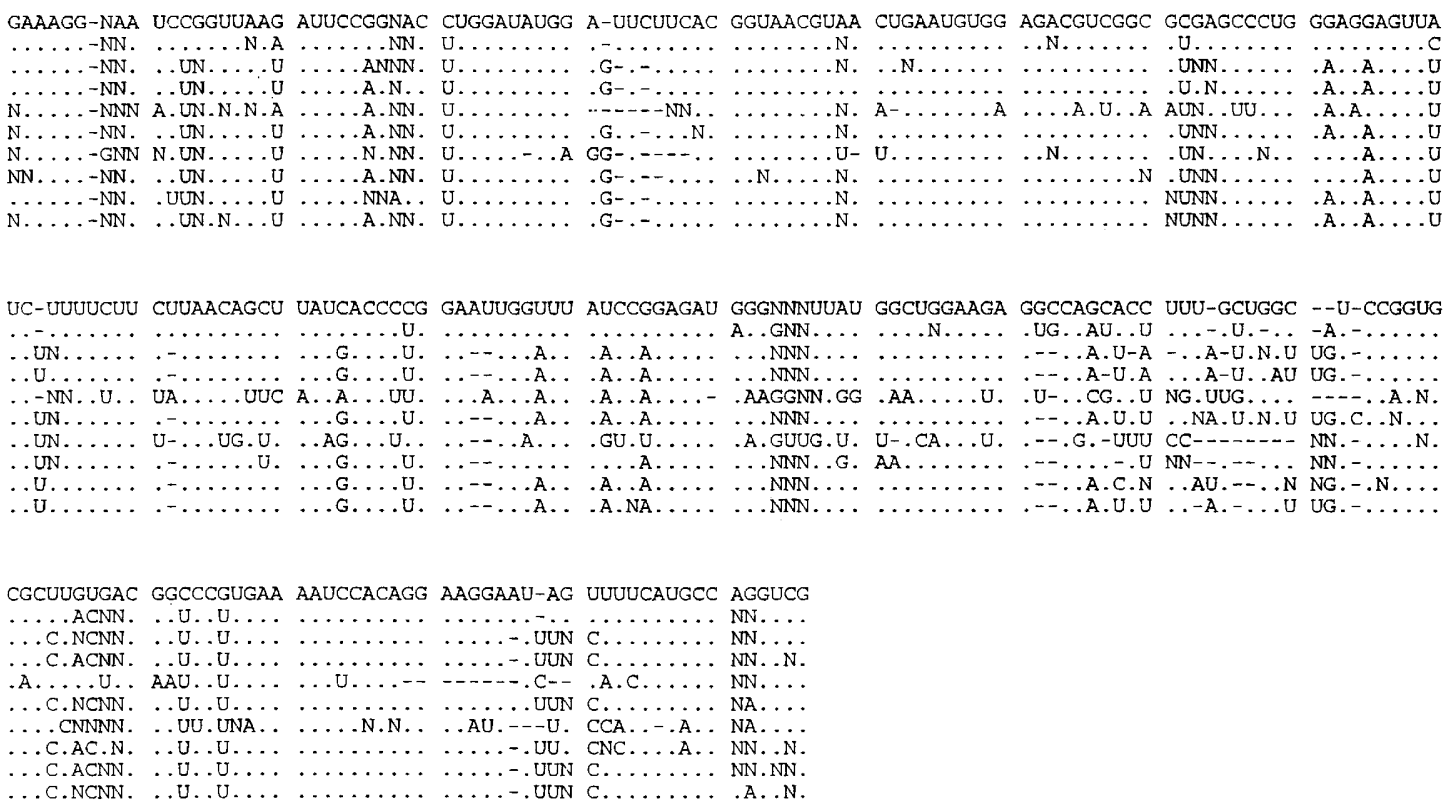

FIG. 2. Alignment of $25 \mathrm{~S}$ rRNA partial sequences initiated at position 1841 from Metschnikowia and reference species. For further explanation see the legend to Fig. 1. 


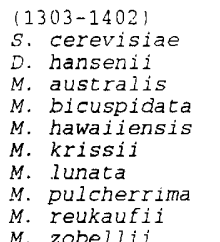

M. zobellii

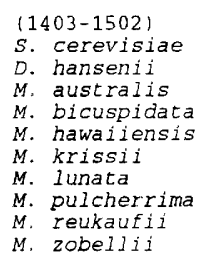

(1503-1596)

$S$. cerevisiae

D. hansenil

$M$. australis

M. bicuspidata

M. hawailensis

M. krissii

M. Iunata

M. pulcherrima

M. reukaufii

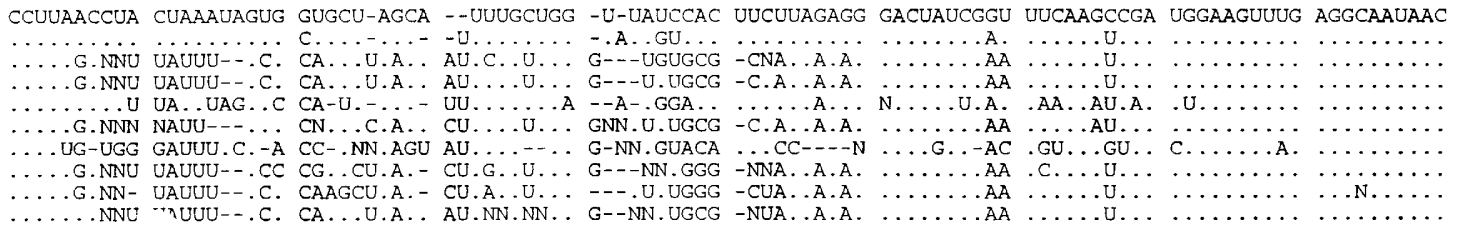

AAGUCUGUGA UGCONUUAGA CGUUCUGGGC NCACGCGCGC UACACUGACG GAGCCAGCGA GU-C-UAACC UUGGCCGAGA GGUCUUGGUA AUCUUGUGAA
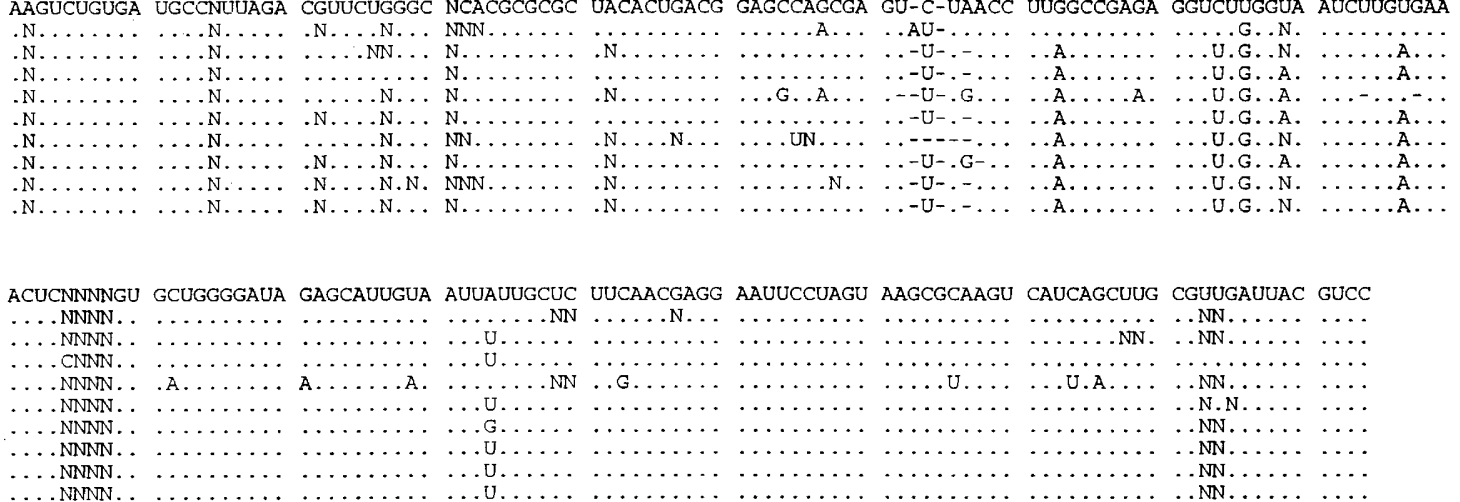

FIG. 3. Alignment of $18 \mathrm{~S}$ rRNA partial sequences initiated at position 1627 from Metschnikowia and reference species. For further explanation see the legend to Fig. 1.

signature nucleotides that separated it from other genera $(11-13,17,22)$. If genera are aggregates of closely related species which arose from a common ancestor, signatures presumably represent conserved nucleotides that reflect this shared phylogeny. Signatures are evident for the genus Metschnikowia if $M$. hawaiiensis is removed, and the genus is even more homogeneous if $M$. lunata is also removed.

Species relationships are shown in Fig. 4. This phylogenetic tree was calculated by the neighbor-joining method. The data set used included all three regions sequenced in order to introduce sufficient nucleotide differences to position closely related species more accurately. The large deletion in region 25S-635 in $M$. hawaiiensis had little impact on the branch lengths of trees generated after this species was removed (data not shown). Table 2 shows that $M$. hawaiiensis is also highly divergent in the other two regions examined. The relationships which were determined indicate that the aquatic Metschnikowia species are separate from the terrestrial species. The low level of detectable DNA-DNA complementarity among aquatic species (19) is consistent with the close relationships revealed by our rRNA comparison.

Bootstrapping of the data suggests that some branch nodes shown in Fig. 4 are weak; for example, a $56 \%$ confidence level was obtained for the $M$. reukaufii branch. Analyses of the data by DNAML and PAUP gave similar overall tree topologies. Both analyses showed that $M$. lunata and $M$. hawaiiensis are outliers and that the latter species is the species that is most distantly related to other members of the genus Metschnikowia. An analysis of individual rRNA regions by the three programs used in this study demonstrated that there is some shifting of species associations. In some treatments of the data, $M$. reukaufii fell in the aquatic species group and $M$. pulcherrima fell at the edge of the aquatic species group. Distances among $M$. lunata, $M$. hawaiiensis, and the reference taxa $(S$. cerevisiae and $D$. hansenii) may

TABLE 2. Matrix of number of nucleotide differences in three rRNA regions for Metschnikowia species and the reference taxa $S$. cerevisiae and D. hansenii

\begin{tabular}{|c|c|c|c|c|c|c|c|c|c|}
\hline \multirow[b]{2}{*}{ Species } & \multicolumn{9}{|c|}{ No. of differences in regions $25 \mathrm{~S}-635,25 \mathrm{~S}-1841$, and $18 \mathrm{~S}-1627^{a}$} \\
\hline & $\begin{array}{c}S . \\
\text { cerevisiae }\end{array}$ & $\begin{array}{c}D . \\
\text { hansenii }\end{array}$ & $\begin{array}{c}M . \\
\text { australis }\end{array}$ & $\begin{array}{c}\text { M. } \\
\text { bicuspidata }\end{array}$ & $\begin{array}{l}M . \\
\text { hawaiiensis }\end{array}$ & $\begin{array}{c}\text { M. } \\
\text { krisii }\end{array}$ & $\begin{array}{c}\text { M. } \\
\text { lunata }\end{array}$ & $\begin{array}{c}\text { M. } \\
\text { pulcherrima }\end{array}$ & $\begin{array}{c}M . \\
\text { reukaufit }\end{array}$ \\
\hline D. hansenii & $57,18,12$ & & & & & & & & \\
\hline M. australis & $114,41,42$ & $103,35,38$ & & & & & & & \\
\hline M. bicuspidata & $106,42,41$ & $99,35,38$ & $24,4,2$ & & & & & & \\
\hline M. hawaiiensis & $164,76,49$ & $156,72,39$ & $111,70,50$ & $119,71,50$ & & & & & \\
\hline M. krissii & $110,37,36$ & $100,30,32$ & $35,7,8$ & $35,7,6$ & $119,70,49$ & & & & \\
\hline M. lunata & $127,71,56$ & $118,66,52$ & $56,53,39$ & $61,54,39$ & $92,87,64$ & $60,55,39$ & & & \\
\hline M. pulcherrima & $113,39,44$ & $108,35,40$ & $37,19,11$ & $45,20,11$ & $109,68,48$ & $51,18,14$ & $47,43,42$ & & \\
\hline M. reukaufii & $103,38,44$ & $96,33,40$ & $39,9,11$ & $31,9,11$ & $113,68,53$ & $35,8,12$ & $59,53,45$ & $40,14,12$ & \\
\hline M. zobellii & $104,39,36$ & $95,34,34$ & $29,6,2$ & $27,6,2$ & $113,68,47$ & $32,4,7$ & $64,52,37$ & $46,14,11$ & $35,5,9$ \\
\hline
\end{tabular}

${ }^{a}$ For each comparison the first number is the number of nucleotide differences in region $25 S-635$, the second number is the number of nucleotide differences in region 25S-1841, and the third number is the number of nucleotide differences in region 18S-1627. The following numbers of nucleotides were compared: region 25S-635, 300 nucleotides; region 25S-1841, 256 nucleotides; and region 18S-1627, 294 nucleotides. Deleted areas were included in these calculations. 


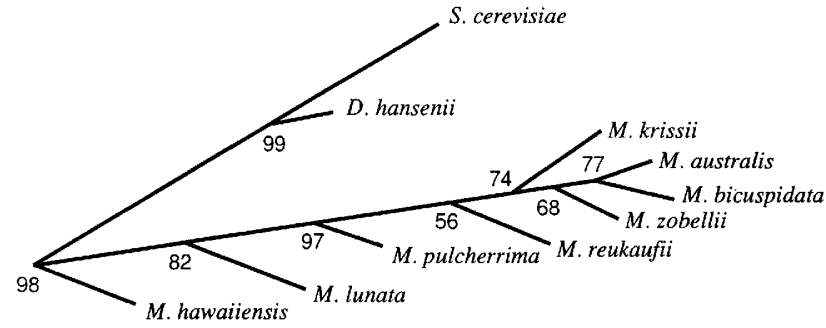

FIG. 4. Phylogenetic tree calculated for species of the genus Metschnikowia and reference taxa by using the neighbor-joining method. The numbers at the branch nodes are frequencies in a set of 100 trees generated by bootstrapping. Branch lengths were derived from an analysis of the consensus tree data by using the program PAUP, version 2.4.1, and are proportional to the observed differences in nucleotides.

have been underestimated because the large number of nucleotide differences shown in Table 2 suggest that mutable sites were saturated and may have undergone multiple substitutions (23).

The extent of rRNA sequence diversity among Metschnikowia species is surprising considering the relative phenotypic homogeneity of these taxa. This suggests that the genus is very old or that it underwent rapid evolution. Since our phylogenetic trees are unrooted, the direction of evolution cannot be deduced. The parasitic associations observed for many of the species could have resulted in greater selective pressure to adapt to the host's immune response in highly specific niches; however, the pressure was apparently low with regard to physiological profiles and ascospore morphology since there is little intrageneric variation in these characteristics (14). The large ascospore size of $M$. hawaiiensis and the curved cell shape of $M$. lunata seem to coincide with the phylogenetic distances of these taxa from the other Metschnikowia species. Inclusion of $M$. hawaiiensis and perhaps $M$. lunata in the genus Metschnikowia is questionable, but possible reassignment to a new genus should await a better understanding of the significance of the rRNA sequence data.

\section{ACKNOWLEDGMENTS}

We thank Christie J. Robnett for excellent technical assistance and S. W. Peterson for assistance with computations.

L.C.M. and A.N.H. are grateful for research fellowships received from Conselho Nacional de Desenvolvimento Cientifico e Tecnologico, Brazil.

\section{REFERENCES}

1. Chirgwin, J. M., A. E. Przybyla, R. J. MacDonald, and W. J. Rutter. 1979. Isolation of biologically active ribonucleic acid from sources enriched in ribonuclease. Biochemistry 18:5284 5299.

2. Fell, J. W., and I. L. Hunter. 1968. Isolation of heterothallic yeast strains of Metschnikowia Kamienski and their mating reaction with Chlamydozyma Wickerham. Antonie van Leeuwenhoek 34:365-376.

3. Fell, J. W., and C. P. Kurtzman. 1990. Nucleotide sequence analysis of a variable region of the large subunit rRNA for identification of marine-occurring yeasts. Curr. Microbiol. 21: 295-300.

4. Fell, J. W., and J. I. Pitt. 1969. Taxonomy of the yeast genus Metschnikowia. J. Bacteriol. 98:853-854.

5. Georgiev, O. I., N. Nikolaev, A. A. Hadjiolov, K. G. Skryabin, V. M. Zakharyev, and A. A. Bayev. 1981. The structure of the yeast ribosomal RNA genes. IV. Complete sequence of the
rRNA gene from Saccharomyces cerevisiae. Nucleic Acids Res. 9:6953-6958.

6. Golubev, W. I. 1977. Metschnikowia lunata sp. nov. Antonie van Leeuwenhoek 43:317-322.

7. Grüss, J. 1918. Uber die Anpassung eines Pilzes (Anthomyces reukaufii) an den Blütenbau und den Bienenrüssel. Ber. Dtsch. Bot. Ges. 35:746-761.

8. Guého, E., C. P. Kurtzman, and S. W. Peterson. 1990. Phylogenetic relationships among species of Sterigmatomyces and Fellomyces as determined from partial rRNA sequences. Int. J. Syst. Bacteriol. 40:60-65.

9. Kamienski, T. 1899. Notice préliminaire sur la nouvelle espèce de Metschnikowia. (Monospora Metschn.). Trav. Soc. Imp. Nat. S. Petersb. 30:363-364.

10. Kurtzman, C. P. 1987. Prediction of biological relatedness among yeasts from comparisons of nuclear DNA complementarity. Stud. Mycol. 30:459-468.

11. Kurtzman, C. P. 1992. rRNA sequence comparisons for assessing phylogenetic relationships among yeasts. Int. J. Syst. Bacteriol. 42:1-6.

11a.Kurtzman, C. P. Unpublished data.

12. Kurtzman, C. P., and Z. Liu. 1990. Evolutionary affinities of species assigned to Lipomyces and Myxozyma estimated from ribosomal RNA sequence divergence. Curr. Microbiol. 21:387393.

13. Kurtzman, C. P., and C. J. Robnett. 1991. Phylogenetic relationships among species of Saccharomyces, Schizosaccharomyces, Debaryomyces, and Schwanniomyces determined from partial rRNA sequences. Yeast 7:61-72.

14. Lachance, M. A., W. T. Starmer, and H. J. Phaff. 1990. Metschnikowia hawaiiensis sp. nov., a heterothallic haploid yeast from Hawaiian morning glory and associated drosophilids. Int. J. Syst. Bacteriol. 40:415-420.

15. Lane, D. J., B. Pace, G. J. Olsen, D. A. Stahl, M. L. Sogin, and N. R. Pace. 1985. Rapid determination of 16 S ribosomal RNA sequences for phylogenetic analysis. Proc. Natl. Acad. Sci. USA 82:6955-6959.

16. Lindner, P. 1901. Mikroskopische Betriebskontrolle in den Gärungsgewerben, 3rd ed. P. Parey, Berlin.

17. Liu, Z., and C. P. Kurtzman. 1991. Phylogenetic relationships among species of Willopsis and Saturnospora gen. nov. as determined from partial rRNA sequences. Antonie van Leeuwenhoek 60:21-30.

18. Mankin, A. S., K. G. Skryabin, and P. M. Rubstov. 1986. Identification of 10 additional nucleotides in the primary structure of yeast 18S RNA. Gene 44:143-145.

19. Mendonça-Hagler, L. C., A. N. Hagler, H. J. Phaff, and J. Tredick. 1985. DNA relatedness among aquatic yeasts of the genus Metschnikowia and proposal of the species Metschnikowia australis comb. nov. Can. J. Microbiol. 31:905-909.

20. Metschnikoff, E. 1884. Über eine Sprosspilzkrankheit der Daphnien. Beitrag zur Lehre über den Kampf der Phagocyten gegen Krankheitserreger. Arch. Pathol. Anat. Physiol. R. Virchow. 96:177-195.

21. Miller, M. W., and H. J. Phaff. 1984. Metschnikowia Kamienski, p. 266-278. In N. J. W. Kreger-van Rij (ed.), The yeasts: a taxonomic study, 3rd ed. Elsevier Science Publishers, Amsterdam.

22. Peterson, S. W., and C. P. Kurtzman. 1990. Phylogenetic relationships among species of the genus Issatchenkia Kudriavzev. Antonie van Leeuwenhoek 58:235-240.

23. Peterson, S. W., and C. P. Kurtzman. 1991. Ribosomal RNA sequence divergence among sibling species of yeasts. Syst. Appl. Microbiol. 14:124-129.

24. Pitt, J. I., and M. W. Miller. 1968. Sporulation in Candida pulcherrima, Candida reukaufii, and Chlamydozyma species and their relationship with Metschnikowia. Mycologia 60:663685.

25. Pitt, J. I., and M. W. Miller. 1970. Speciation in the yeast genus Metschnikowia. Antonie van Leeuwenhoek 36:357-381.

26. Rubstov, P. M., M. M. Musakhanov, V. M. Zakharyev, A. S. Krayev, K. G. Skryabin, and A. A. Bayev. 1980. The complete structure of yeast ribosomal RNA genes. I. The complete 
nucleotide sequence of the $18 \mathrm{~S}$ ribosomal RNA gene from Saccharomyces cerevisiae. Nucleic Acids Res. 8:758-762.

27. Sanger, F., S. Nicklen, and A. R. Coulson. 1977. DNA sequencing with chain-terminating inhibitors. Proc. Natl. Acad. Sci. USA 74:5463-5467.

28. Spencer, J. F. T., H. J. Phaff, and N. R. Gardner. 1964. Metschnikowia kamienskii sp. n., a yeast associated with brine shrimp. J. Bacteriol. 88:758-762.

29. Timberlake, W. E. 1978. Low repetitive DNA content in Aspergillus nidulans. Science 202:973-975.

30. van Uden, N., and R. Castelo-Branco. 1961. Metschnikowia zobellii sp. nov. and Metschnikowia krissii sp. nov., two yeasts from the Pacific Ocean pathogenic for Daphnia magna. J. Gen. Microbiol. 26:141-148.

31. Wickerham, L. J. 1951. Taxonomy of the yeasts. U.S. Dep.
Agric. Tech. Bull. 1029:1-56.

32. Wickerham, L. J. 1964. A preliminary report on a perfect family of exclusively protosexual yeasts. Mycologia 54:253-266.

33. Woese, C. R. 1987. Bacterial evolution. Microbial Rev. 51:221271.

34. Yamada, Y., and Y. Nakagawa. 1990. The molecular phylogeny of the basidiomycetous yeast species Leucosporidium scottii based on the partial sequences of $18 \mathrm{~S}$ and $26 \mathrm{~S}$ ribosomal ribonucleic acids. J. Gen. Appl. Microbiol. 36:63-68.

35. Yamada, Y., Y. Nakagawa, T. Nakase, and I. Banno. 1989. The phylogenetic relationship of the conidium-forming anamorphic yeast genera Sterigmatomyces, Kurtzmanomyces, Tsuchiyaea and Fellomyces, and the teleomorphic yeast genus Sterigmatosporidium on the basis of the partial sequences of $18 \mathrm{~S}$ and 26S ribosomal ribonucleic acids. Agric. Biol. Chem. 53:53-68. 